\title{
Besov regularity for a class of singular or degenerate elliptic equations
}

\author{
Pasquale Ambrosio
}

September 1, 2021

\begin{abstract}
Motivated by applications to congested traffic problems, we establish higher integrability results for the gradient of local weak solutions to the strongly degenerate or singular elliptic PDE

$$
-\operatorname{div}\left((|\nabla u|-1)_{+}^{q-1} \frac{\nabla u}{|\nabla u|}\right)=f, \quad \text { in } \Omega,
$$

where $\Omega$ is a bounded domain in $\mathbb{R}^{n}$ for $n \geq 2,1<q<\infty$ and $(\cdot)_{+}$stands for the positive part. We assume that the datum $f$ belongs to a suitable Sobolev or Besov space. The main novelty here is that we deal with the case of subquadratic growth, i.e. $1<q<2$, which has so far been neglected. In the latter case, we also prove the higher fractional differentiability of the solution to a variational problem, which is characterized by the above equation. For the sake of completeness, we finally give a Besov regularity result also in the case $q \geq 2$.
\end{abstract}

AMS Classification: 35B65, 35J70, 35J75, 49N60.

Keywords: Singular elliptic equations; degenerate elliptic equations; higher integrability; Besov spaces.

\section{Introduction}

Let us consider the following pair of variational problems in duality:

$$
\inf _{\sigma \in L^{p}\left(\Omega, \mathbb{R}^{n}\right)}\left\{\int_{\Omega} \mathcal{H}(\sigma(x)) d x:-\operatorname{div} \sigma=f,\langle\sigma, \nu\rangle=0 \text { on } \partial \Omega\right\}
$$

and

$$
\sup _{u \in W^{1, p^{\prime}}(\Omega)}\left\{\int_{\Omega} u(x) f(x) d x-\int_{\Omega} \mathcal{H}^{*}(\nabla u(x)) d x\right\},
$$

where $\Omega$ is a bounded connected open subset of $\mathbb{R}^{n}(n \geq 2)$ with Lipschitz boundary, $f$ is a given function defined over $\bar{\Omega}$ with zero mean (i.e. $\int_{\Omega} f \overline{d x}=0$ ) and the function $\mathcal{H}$ is defined 
for all $\sigma \in \mathbb{R}^{n}$ by

$$
\mathcal{H}(\sigma):=\frac{1}{p}|\sigma|^{p}+|\sigma|
$$

where $p \in(1,+\infty)$. With such a choice, we get

$$
\nabla \mathcal{H}^{*}(z)=(|z|-1)_{+}^{q-1} \frac{z}{|z|}, \quad z \in \mathbb{R}^{n}
$$

where $\mathcal{H}^{*}$ is the Legendre transform of $\mathcal{H}, q=p^{\prime}=p /(p-1)$ is the conjugate exponent of $p$ and $(\cdot)_{+}$stands for the positive part (see [9]). It is well known that the Euler-Lagrange equation of the functional in $(\overline{\mathrm{P} 2})$ is given by the $\mathrm{PDE}$

$$
\left\{\begin{array}{rc}
-\operatorname{div}\left(\nabla \mathcal{H}^{*}(\nabla u)\right)=f & \text { in } \Omega, \\
\left\langle\nabla \mathcal{H}^{*}(\nabla u), \nu\right\rangle=0 & \text { on } \partial \Omega,
\end{array}\right.
$$

which has to be meant in the distributional sense.

In this paper we prove local higher integrability results both for the solution to (P1) and for the gradient of the local weak solutions to the equation appearing in (1.2), when the datum $f$ belongs either to the Sobolev class $W_{l o c}^{1, p}(\Omega)$ or to the local Besov space $B_{p, \infty, l o c}^{\alpha}(\Omega)$, for some $\alpha \in(0,1)$. Furthermore, we establish the higher fractional differentiability of the solution to (P1) in the case $1<q<2$, under the same assumptions about the datum $f$.

From the very beginning we warn the reader that, for the sake of simplicity, we will confine ourselves to the case of the cost function $\mathcal{H}$ defined in (1.1), rather than considering the more general function

$$
\sigma \in \mathbb{R}^{n} \mapsto \frac{1}{p}|\sigma|^{p}+\delta|\sigma|
$$

which leads to the equation

$$
-\operatorname{div}\left((|\nabla u|-\delta)_{+}^{q-1} \frac{\nabla u}{|\nabla u|}\right)=f
$$

where $\delta>0$. Indeed, there is no loss of generality in supposing $\delta=1$ and our proofs still work in the more general case (1.3), with minor modifications.

If we suppose that $f \in L^{p}(\Omega)$ and that the infimum in (P1) is finite, then problem (P1) consists in minimizing a strictly convex and coercive functional on $L^{p}$ subject to a convex and closed constraint: therefore, a solution $\sigma_{0}$ to $(\underline{\mathrm{P} 1})$ exists and must be unique. Moreover, we recall that by standard convex duality (see [14] for example), the values inf ( $\overline{\text { P1 }}$ ) and sup (Iי) coincide and the primal-dual optimality condition characterizes the minimizer $\sigma_{0}$ of (P1) through the equality

$$
\sigma_{0}(x)=\nabla \mathcal{H}^{*}\left(\nabla u_{0}(x)\right), \quad \text { for } \mathscr{L}^{n} \text {-a.e. } x \in \Omega,
$$

where $u_{0}$ is a solution of $(\overline{\mathrm{P} 2})$ and $\mathscr{L}^{n}$ denotes the $n$-dimensional Lebesgue measure. This is equivalent to the requirement that $u_{0}$ is a weak solution of the Euler-Lagrange equation (1.2), in the sense that

$$
\int_{\Omega}\left\langle\nabla \mathcal{H}^{*}\left(\nabla u_{0}(x)\right), \nabla \varphi(x)\right\rangle d x=\int_{\Omega} f(x) \varphi(x) d x, \quad \text { for every } \varphi \in W^{1, q}(\Omega) .
$$

Furthermore, since $f$ has zero mean, using the direct methods of the Calculus of Variations it is not difficult to show that the dual problem ( $(\underline{\mathrm{P} 2})$ admits at least one solution $u_{0}$ belonging to 
$W_{\diamond}^{1, q}(\Omega)$, where

$$
W_{\diamond}^{1, q}(\Omega):=\left\{u \in W^{1, q}(\Omega): \int_{\Omega} u(x) d x=0\right\},
$$

and such that (1.4) holds, so that $u_{0}$ is a distributional solution of the strongly degenerate or singular elliptic equation

$$
-\operatorname{div}\left((|\nabla u|-1)_{+}^{q-1} \frac{\nabla u}{|\nabla u|}\right)=f,
$$

under homogeneous Neumann boundary conditions. We also note that, in general, if one looks at the solutions $u$ of the above equation, no more than $C^{0,1}$ regularity should be expected for them: indeed, every 1-Lipschitz function $u$ is a solution of the homogeneous equation. Moreover, when $q \geq 2$ we have

$$
\frac{(|\nabla u|-1)_{+}^{q-1}}{|\nabla u|}|\xi|^{2} \leq\left\langle D^{2} \mathcal{H}^{*}(\nabla u) \xi, \xi\right\rangle \leq(q-1)(|\nabla u|-1)_{+}^{q-2}|\xi|^{2}, \quad \xi \in \mathbb{R}^{n},
$$

that is, the Hessian matrix $D^{2} \mathcal{H}^{*}$ has eigenvalues becoming degenerate in the region $\{|\nabla u| \leq 1\}$.

The main novelty of this paper is that we shall address the singular case $1<q<2$, which has so far been neglected, since extra technical difficulties arise concerning elliptic regularity whenever we are in this case. This phenomenon, which also occurs in the classical $q$-harmonic setting, has been very well explained in [4, Section 2.6].

The regularity properties of the solutions to PDEs as the one in (1.5) have been widely investigated. Actually, as we have already said, these PDEs can be considered as the EulerLagrange equation of the functional appearing in (IP2)

$$
\mathcal{F}(u, \Omega)=\int_{\Omega} \frac{1}{q}(|\nabla u|-1)_{+}^{q} d x-\int_{\Omega} u f d x,
$$

which fits into the broader context of functionals having a q-Laplacian type structure at infinity. The local Lipschitz regularity for the minimizers of asymptotically convex problems has been proved when $f=0$ under quadratic growth assumptions in [10], and later in [19] for superquadratic growth conditions and in [22] in the case of subquadratic growth (see also [6] and [17]). In addition, similar results have been obtained for functionals whose integrands also depend on lower-order terms (see [15, 16, 23] and [24] for example).

A further topic concerning the regularity of solutions to equations as the one in (1.5) is the investigation of their higher differentiability of both integer and fractional order, and several results are available so far (see, for example, [3, 12] and [18]). In this regard, it must be mentioned that to the best of our knowledge, different authors have proved the (local) higher differentiability of integer order of a certain function of the gradient of the solutions to (1.5) that takes into account the degeneracy of the equation in the case $q \geq 2$ and $f \in W_{l o c}^{1, p}(\Omega)$ : for further information see [7, 9, 11], as well as [13] for the case $f=0$.

The technical difficulties encountered in dealing with the singular case $1<q<2$ prevent us from achieving the same kind of regularity results as those obtained for (P1) and (1.5) by Brasco, Carlier and Santambrogio in [9]: there, they confine their analysis to the non-singular case $q \geq 2$, by assuming that $f \in W^{1, p}(\Omega)$. In particular, they show that if $u \in W^{1, q}(\Omega)$ is a local weak solution of (1.5), then $\sigma_{0}=\nabla \mathcal{H}^{*}(\nabla u) \in W_{l o c}^{1, r}(\Omega)$ for a suitable exponent $r=r(n, q)$. Moreover, using the Sobolev embedding theorems, they find that $\sigma_{0} \in L_{l o c}^{s}(\Omega)$ for all $s \in[1,+\infty)$ when $n=2$. As for the higher integrability of $\nabla u$, they get $\nabla u \in L_{l o c}^{q \frac{n}{n-2}}(\Omega)$ in the case $n>2$, while if $n=2$ they get $\nabla u \in L_{l o c}^{s}(\Omega)$ for all $s \in[1,+\infty)$ (see [9]). 
However, despite the difficulties we have mentioned, we are able to establish the following

Theorem 1.1. Let $n \geq 2, q \in(1,2)$ and $\alpha \in(0,1)$. Moreover, let $u \in W_{\text {loc }}^{1, q}(\Omega)$ be a local weak solution of the equation

$$
-\operatorname{div}\left(\nabla \mathcal{H}^{*}(\nabla u)\right)=f
$$

Then, for the function

$$
H_{\frac{q}{2}}(\nabla u):=(|\nabla u|-1)_{+}^{\frac{q}{2}} \frac{\nabla u}{|\nabla u|}
$$

the following implications hold

$$
\begin{gathered}
f \in W_{l o c}^{1, p}(\Omega) \Rightarrow H_{\frac{q}{2}}(\nabla u) \in B_{2, \infty}^{\frac{1}{3-q}} \text { locally in } \Omega \\
f \in B_{p, \infty, l o c}^{\alpha}(\Omega) \Rightarrow H_{\frac{q}{2}}(\nabla u) \in B_{2, \infty}^{\min \left\{\frac{\alpha+1}{2}, \frac{1}{3-q}\right\}} \text { locally in } \Omega .
\end{gathered}
$$

Furthermore:

(a) if $f \in W_{\text {loc }}^{1, p}(\Omega)$, then for any ball $B_{R} \Subset \Omega$ the following estimate

$$
\int_{B_{R / 2}}\left|\tau_{s, h} H_{\frac{q}{2}}(\nabla u)-H_{\frac{q}{2}}(\nabla u)\right|^{2} d x \leq C_{1}\left(\|\nabla f\|_{L^{p}\left(B^{\prime}\right)}\|\nabla u\|_{L^{q}\left(B^{\prime}\right)}|h|^{2}+\|\nabla u\|_{L^{q}\left(B^{\prime}\right)}^{q}|h|^{\frac{2}{3-q}}\right)
$$

holds true for every $s \in\{1, \ldots, n\}$, for every $h \in \mathbb{R}$ such that $|h| \leq r_{0}<\frac{1}{2} \operatorname{dist}\left(B_{R}, \partial \Omega\right)$, for $B^{\prime}=B_{R}+B\left(0, r_{0}\right)$ and a positive constant $C_{1}=C_{1}(R, q, n)$.

(b) If, on the other hand, $f \in B_{p, \infty, l o c}^{\alpha}(\Omega)$, then for any ball $B_{R} \Subset \Omega$ the following estimate

$$
\int_{B_{R / 2}}\left|\tau_{s, h} H_{\frac{q}{2}}(\nabla u)-H_{\frac{q}{2}}(\nabla u)\right|^{2} d x \leq C_{2}\left(\|f\|_{B_{p, \infty}^{\alpha}\left(B^{\prime}\right)}\|\nabla u\|_{L^{q}\left(B^{\prime}\right)}|h|^{\alpha+1}+\|\nabla u\|_{L^{q}\left(B^{\prime}\right)}^{q}|h|^{\frac{2}{3-q}}\right)
$$

holds true for every $s \in\{1, \ldots, n\}$, for every $h \in \mathbb{R}$ such that $|h| \leq r_{0}<\frac{1}{2} \operatorname{dist}\left(B_{R}, \partial \Omega\right)$, for $B^{\prime}=B_{R}+B\left(0, r_{0}\right)$ and a positive constant $C_{2}=C_{2}(R, q, n)$.

The main tool in the proof of Theorem 1.1 is the difference quotient technique, which, due to the singular character of the case under consideration, requires new ideas with respect to previous papers on the subject ([1] and [26]), where the case $1<q<2$ has been treated, but in the absence of singularity. For the sake of clarity, let us now try to explain the difficulties associated with the singular setting: when dealing with the higher differentiability of solutions to subquadratic problems, the difference quotient method usually yields a Caccioppoli-type inequality based on the possibility of reabsorbing the terms in the right-hand side that involve the increment of the gradient of a solution by the left-hand side. In the singular setting, this possibility is actually excluded by the fact that we cannot control the difference quotient of the gradient of a solution $u$ in terms of $H_{\frac{q}{2}}(\nabla u)$, whose difference quotient appears in the left-hand side, and this is true regardless of the type of regularity of the datum $f$. Actually, even in the case $f=0$, the integral appearing in the right-hand side of formula (3.2) cannot be estimated in a standard way, since the difference quotient $\Delta_{h} u$ cannot be controlled in terms of $\Delta_{h}\left(\nabla \mathcal{H}^{*}(\nabla u)\right)$, unless we perform the estimate far away from the set $\{x:|\nabla u(x)| \leq 1\}$. On the other hand, as we have already observed, every 1-Lipschitz function $u$ is a solution of the 
homogeneous equation

$$
-\operatorname{div}\left(\nabla \mathcal{H}^{*}(\nabla u)\right)=0,
$$

so that a more regular $f$ may not be sufficient to ensure a higher differentiability property of the solutions.

Returning to the variational problem (P1), thanks to Theorem 1.1 we can obtain the following higher differentiability result of fractional order for its (unique) minimizer:

Theorem 1.2. Let $n \geq 2, q \in(1,2)$ and $\alpha \in(0,1)$. Moreover, let $\sigma_{0} \in L^{p}\left(\Omega, \mathbb{R}^{n}\right)$ be the solution of ( $(\mathrm{P1})$. Then, the following implications hold:

$$
\begin{gathered}
f \in W_{l o c}^{1, p}(\Omega) \Rightarrow \sigma_{0} \in B_{p, \infty}^{\frac{2}{p(3-q)}} \text { locally in } \Omega \\
f \in B_{p, \infty, l o c}^{\alpha}(\Omega) \Rightarrow \sigma_{0} \in B_{p, \infty}^{\min \left\{\frac{\alpha+1}{p}, \frac{2}{p(3-q)}\right\}} \text { locally in } \Omega .
\end{gathered}
$$

Furthermore:

(a) if $f \in W_{\text {loc }}^{1, p}(\Omega)$, then for every solution $u$ of problem ( $\mathrm{P2}$ ) and every ball $B_{R} \Subset \Omega$, the following estimate

$$
\int_{B_{R / 2}}\left|\tau_{s, h} \sigma_{0}-\sigma_{0}\right|^{p} d x \leq C_{1}\left(\|\nabla f\|_{L^{p}\left(B^{\prime}\right)}\|\nabla u\|_{L^{q}\left(B^{\prime}\right)}|h|^{2}+\|\nabla u\|_{L^{q}\left(B^{\prime}\right)}^{q}|h|^{\frac{2}{3-q}}\right)
$$

holds true for every $s \in\{1, \ldots, n\}$, for every $h \in \mathbb{R}$ such that $|h| \leq r_{0}<\frac{1}{2} \operatorname{dist}\left(B_{R}, \partial \Omega\right)$, for $B^{\prime}=B_{R}+B\left(0, r_{0}\right)$ and a positive constant $C_{1}=C_{1}(R, q, n)$.

(b) If, on the other hand, $f \in B_{p, \infty, l o c}^{\alpha}(\Omega)$, then for every solution u of problem (P2) and every ball $B_{R} \Subset \Omega$, the following estimate

$$
\int_{B_{R / 2}}\left|\tau_{s, h} \sigma_{0}-\sigma_{0}\right|^{p} d x \leq C_{2}\left(\|f\|_{B_{p, \infty}^{\alpha}\left(B^{\prime}\right)}\|\nabla u\|_{L^{q}\left(B^{\prime}\right)}|h|^{\alpha+1}+\|\nabla u\|_{L^{q}\left(B^{\prime}\right)}^{q}|h|^{\frac{2}{3-q}}\right)
$$

holds true for every $s \in\{1, \ldots, n\}$, for every $h \in \mathbb{R}$ such that $|h| \leq r_{0}<\frac{1}{2} \operatorname{dist}\left(B_{R}, \partial \Omega\right)$, for $B^{\prime}=B_{R}+B\left(0, r_{0}\right)$ and a positive constant $C_{2}=C_{2}(R, q, n)$.

Remark 1.3. We immediately observe that if we take $q=p=2$ and $f \in W_{l o c}^{1,2}(\Omega)$ in the statements $(a)$ of Theorems 1.1 and 1.2, then we get back the full Sobolev regularity results of [9, Theorem 4.2 and Corollary 4.3], i.e. there is no discrepancy between the old and the new results.

As a consequence of the above theorem, using a local version of Sobolev's embedding theorem for Besov spaces, we obtain the following higher integrability result for $\sigma_{0}$ :

Corollary 1.4. Under the assumptions of Theorem 1.2, we obtain the following implications:

$$
\begin{gathered}
f \in W_{l o c}^{1, p}(\Omega) \Rightarrow \sigma_{0} \in L_{l o c}^{r}(\Omega) \text { for all } r \in\left[1, \frac{n p(3-q)}{n(3-q)-2}\right), \\
f \in B_{p, \infty, l o c}^{\alpha}(\Omega) \Rightarrow \sigma_{0} \in L_{l o c}^{s}(\Omega),
\end{gathered}
$$


where

$$
s= \begin{cases}\text { any value }<\frac{n p}{n-\alpha-1}, & \text { if } 0<\alpha<\frac{q-1}{3-q}, \\ \text { any value }<\frac{n p(3-q)}{n(3-q)-2}, & \text { if } \frac{q-1}{3-q} \leq \alpha<1 .\end{cases}
$$

Finally, as an easy consequence of the previous results, we also get a gain of integrability for the gradient of the local weak solutions of (1.6). Indeed, we have the following

Corollary 1.5. Under the assumptions of Theorem 1.1, we obtain the following implications:

$$
\begin{gathered}
f \in W_{l o c}^{1, p}(\Omega) \Rightarrow \nabla u \in L_{l o c}^{r}(\Omega) \text { for all } r \in\left[1, \frac{n q(3-q)}{n(3-q)-2}\right), \\
f \in B_{p, \infty, l o c}^{\alpha}(\Omega) \Rightarrow \nabla u \in L_{l o c}^{s}(\Omega),
\end{gathered}
$$

where

$$
s= \begin{cases}\text { any value }<\frac{n q}{n-\alpha-1}, & \text { if } 0<\alpha<\frac{q-1}{3-q}, \\ \text { any value }<\frac{n q(3-q)}{n(3-q)-2}, & \text { if } \frac{q-1}{3-q} \leq \alpha<1 .\end{cases}
$$

For the sake of completeness, in this paper we shall prove that similar results also hold in the case $q \geq 2$, by assuming that $f \in B_{p, \infty, l o c}^{\alpha}(\Omega)$ for some $\alpha \in(0,1)$. Actually, on the one hand, Theorem 4.1 below extends the results proved in [12] to a widely degenerate setting; on the other hand, it extends the aforementioned results contained in [9] to the case of data in a local Besov space.

It is worth mentioning that if $f \in W_{\diamond}^{1, p}(\Omega)$, then for suitable values of $n \geq 2$ and $p>2$ there are some cases in which it is possible to prove that every solution $u \in W_{\diamond}^{1, q}(\Omega)$ of the Neumann boundary problem (1.2) belongs to $W^{1, \infty}(\Omega)$ (see [6, Theorem 2.1]). However, in these cases, the boundedness of $\nabla u$ gives neither a weak differentiability of integer order for $H_{\frac{q}{2}}(\nabla u)$ nor a fractional differentiability of higher order than that obtained in Theorem 1.1.

Before describing the structure of this paper, we wish to briefly motivate what follows by

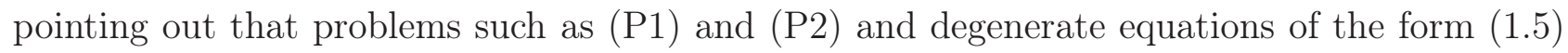
may arise in optimal transport problems with congestion effects on very dense two-dimensional networks. These problems lead, at the limit, to minimization problems posed on measures on curves, as shown in [2]. In recent works ([7] and [9] again), Brasco, Carlier and Santambrogio have gone one step further, by showing that these latter problems can be reformulated in terms of the minimization of an integral functional over a set of vector fields with prescribed divergence, exactly as in problem (P1), which was introduced by Beckmann in the 1950s (see [5]). For further details, we refer the interested reader to [6, Sections 1-2] and [8, Section 1].

The paper is organized as follows. Section 2 is devoted to the preliminaries: after a list of some classical notations and some essential estimates, we recall the basic properties of BesovLipschitz spaces and some elementary properties of the difference quotients of Sobolev functions. In Section 3, we prove Theorems 1.1 and 1.2 and Corollaries 1.4 and 1.5, Finally, in Section 4 we give similar results in the non-singular case $q \geq 2$. 


\section{Notations and preliminaries}

In this paper we shall denote by $C$ or $c$ a general positive constant that may vary on different occasions. Relevant dependencies on parameters and special constants will be suitably emphasized using parentheses or subscripts. The norm we use on $\mathbb{R}^{n}$ will be the standard Euclidean one and it will be denoted by $|\cdot|$. In particular, for the vectors $\xi, \eta \in \mathbb{R}^{n}$, we write $\langle\xi, \eta\rangle$ for the usual inner product and $|\xi|:=\langle\xi, \xi\rangle^{\frac{1}{2}}$ for the corresponding Euclidean norm.

In what follows, $B(x, r)=B_{r}(x)=\left\{y \in \mathbb{R}^{n}:|y-x|<r\right\}$ will denote the ball centered at $x$ of radius $r$. We shall omit the dependence on the center and on the radius when no confusion arises.

We now recall some tools that will be useful to prove our results. For the auxiliary function $V_{\gamma}$, defined for all $\xi \in \mathbb{R}^{n}$ as

$$
V_{\gamma}(\xi):=\left(\mu^{2}+|\xi|^{2}\right)^{\gamma / 2} \xi
$$

where $\mu \geq 0$ and $\gamma>0$ are parameters, we record the following estimates (see [19]):

Lemma 2.1. Let $\gamma>0$ and $\mu \geq 0$. Then there exist two positive constants $c_{1}(\gamma)$ and $c_{2}(\gamma)$ such that

$$
c_{1}(\gamma)|\xi-\eta| \leq \frac{\left|V_{\gamma}(\xi)-V_{\gamma}(\eta)\right|}{\left(\mu^{2}+|\xi|^{2}+|\eta|^{2}\right)^{\gamma / 2}} \leq c_{2}(\gamma)|\xi-\eta|
$$

for any $\xi, \eta \in \mathbb{R}^{n}$.

The next result is an extension of the previous lemma to the case $\gamma \in(-1,0)$ (see the proof of [1, Lemma 2.2]):

Lemma 2.2. For every $\gamma \in(-1 / 2,0)$ and $\mu \geq 0$ we have

$$
(2 \gamma+1)|\xi-\eta| \leq \frac{\left|\left(\mu^{2}+|\xi|^{2}\right)^{\gamma} \xi-\left(\mu^{2}+|\eta|^{2}\right)^{\gamma} \eta\right|}{\left(\mu^{2}+|\xi|^{2}+|\eta|^{2}\right)^{\gamma}} \leq \frac{c(n)}{2 \gamma+1}|\xi-\eta|
$$

for any $\xi, \eta \in \mathbb{R}^{n}$. Moreover, setting

$$
F(\zeta):=\frac{1}{2(\gamma+1)}\left(\mu^{2}+|\zeta|^{2}\right)^{\gamma+1}, \quad \zeta \in \mathbb{R}^{n}
$$

we obtain

$$
\nabla F(\zeta)=\left(\mu^{2}+|\zeta|^{2}\right)^{\gamma} \zeta, \quad \zeta \in \mathbb{R}^{n}
$$

and

$$
\langle\nabla F(\xi)-\nabla F(\eta), \xi-\eta\rangle \geq(2 \gamma+1)\left(\mu^{2}+|\xi|^{2}+|\eta|^{2}\right)^{\gamma}|\xi-\eta|^{2}
$$

for any $\xi, \eta \in \mathbb{R}^{n}$.

In the following, we shall also use the auxiliary function $H_{\alpha}: \mathbb{R}^{n} \rightarrow \mathbb{R}^{n}$ defined as

$$
H_{\alpha}(\xi):=(|\xi|-1)_{+}^{\alpha} \frac{\xi}{|\xi|}
$$

where $\alpha>0$ is a parameter. As for the above function, we can obtain the following result that will be needed to prove Theorem 1.1 : 
Lemma 2.3. For every $(\alpha, \varepsilon) \in \mathbb{R}^{+} \times \mathbb{R}^{+}$with $\alpha<\varepsilon$, there exist two positive constants $\beta_{1}(\alpha, \varepsilon)$ and $\beta_{2}(\alpha, \varepsilon, n)$ such that

$$
\beta_{1}\left|H_{\varepsilon}(\xi)-H_{\varepsilon}(\eta)\right| \leq \frac{\left|H_{\alpha}(\xi)-H_{\alpha}(\eta)\right|}{\left((|\xi|-1)_{+}^{\varepsilon}+(|\eta|-1)_{+}^{\varepsilon}\right)^{\frac{\alpha-\varepsilon}{\varepsilon}}} \leq \beta_{2}\left|H_{\varepsilon}(\xi)-H_{\varepsilon}(\eta)\right|
$$

for any $\xi, \eta \in \mathbb{R}^{n}$.

Proof. By Lemma 2.2, we have that for every $\gamma \in(-1,0)$ there exist two positive constants $k_{1}(\gamma)$ and $k_{2}(\gamma, n)$ such that

$$
k_{1}|z-w|^{2} \leq \frac{\left.|| z\right|^{\gamma} z-\left.|w|^{\gamma} w\right|^{2}}{\left(|z|^{2}+|w|^{2}\right)^{\gamma}} \leq k_{2}|z-w|^{2}
$$

for any $z, w \in \mathbb{R}^{n}$. Now we recall that for every $\gamma \in(-1,0)$ and every $z, w \in \mathbb{R}^{n}$ we get

$$
\frac{1}{\left(|z|^{2}+|w|^{2}\right)^{\gamma}} \leq \frac{1}{(|z|+|w|)^{2 \gamma}} \quad \text { and } \quad \frac{1}{\left(|z|^{2}+|w|^{2}\right)^{\gamma}} \geq \frac{2^{\gamma}}{(|z|+|w|)^{2 \gamma}}
$$

From this observation and from (2.4), it follows that for every $\gamma \in(-1,0)$ there exist two positive constants $k_{3}(\gamma)$ and $k_{4}(\gamma, n)$ such that

$$
k_{3}|z-w| \leq \frac{\left.|| z\right|^{\gamma} z-|w|^{\gamma} w \mid}{(|z|+|w|)^{\gamma}} \leq k_{4}|z-w|
$$

for any $z, w \in \mathbb{R}^{n}$. Then, for any fixed $(\alpha, \varepsilon) \in \mathbb{R}^{+} \times \mathbb{R}^{+}$such that $\alpha<\varepsilon$, by taking

$$
z=H_{\varepsilon}(\xi), \quad w=H_{\varepsilon}(\eta) \text { and } \quad \gamma=\frac{\alpha-\varepsilon}{\varepsilon}
$$

into (2.5), we obtain the desired conclusion.

Now we recall a result that will be needed to prove Theorem 4.1 and whose proof can be found in [9, Lemma 4.1] :

Lemma 2.4. If $2 \leq q<\infty$, then for every $\xi, \eta \in \mathbb{R}^{n}$ we get

$$
\begin{gathered}
\left\langle H_{q-1}(\xi)-H_{q-1}(\eta), \xi-\eta\right\rangle \geq \frac{4}{q^{2}}\left|H_{\frac{q}{2}}(\xi)-H_{\frac{q}{2}}(\eta)\right|^{2} \\
\left|H_{q-1}(\xi)-H_{q-1}(\eta)\right| \leq(q-1)\left(\left|H_{\frac{q}{2}}(\xi)\right|^{\frac{q-2}{q}}+\left|H_{\frac{q}{2}}(\eta)\right|^{\frac{q-2}{q}}\right)\left|H_{\frac{q}{2}}(\xi)-H_{\frac{q}{2}}(\eta)\right| .
\end{gathered}
$$

For further needs, we shall now prove that the first estimate of the previous lemma holds true also in the case $1<q<2$. More precisely, we have the following

Lemma 2.5. If $1<q<2$, then there exists a constant $\beta \equiv \beta(q, n)>0$ such that

$$
\left\langle H_{q-1}(\xi)-H_{q-1}(\eta), \xi-\eta\right\rangle \geq \beta\left|H_{\frac{q}{2}}(\xi)-H_{\frac{q}{2}}(\eta)\right|^{2}
$$

for every $\xi, \eta \in \mathbb{R}^{n}$. 
Proof. We first note that the above inequality is trivially satisfied when $|\xi|,|\eta| \leq 1$. Taking $\gamma=(q-2) / 2$ and $\mu=0$, equality (2.2) becomes

$$
\nabla F(\zeta)=|\zeta|^{q-2} \zeta, \quad \zeta \in \mathbb{R}^{n}
$$

and estimates (2.3) and (2.1) imply

$$
\begin{aligned}
\left\langle|z|^{q-2} z-|w|^{q-2} w, z-w\right\rangle & \geq(q-1)\left(|z|^{2}+|w|^{2}\right)^{\frac{q-2}{2}}|z-w|^{2} \\
& \geq\left.\beta(q, n)|| z\right|^{\frac{q-2}{2}} z-\left.|w|^{\frac{q-2}{2}} w\right|^{2}
\end{aligned}
$$

for any $z, w \in \mathbb{R}^{n}$. Taking $z=(|\xi|-1)_{+} \xi /|\xi|$ and $w=(|\eta|-1)_{+} \eta /|\eta|$ into (2.6), we obtain

$$
\left\langle H_{q-1}(\xi)-H_{q-1}(\eta),(|\xi|-1)_{+} \frac{\xi}{|\xi|}-(|\eta|-1)_{+} \frac{\eta}{|\eta|}\right\rangle \geq \beta(q, n)\left|H_{\frac{q}{2}}(\xi)-H_{\frac{q}{2}}(\eta)\right|^{2} .
$$

Now let $\xi, \eta \in \mathbb{R}^{n}$ be such that $|\xi|,|\eta|>1$ and write the left-hand side of the previous inequality as the difference of two terms $A-B$, where

$$
A:=\left\langle H_{q-1}(\xi)-H_{q-1}(\eta), \xi-\eta\right\rangle
$$

and

$$
B:=\left\langle H_{q-1}(\xi)-H_{q-1}(\eta), \xi-(|\xi|-1)_{+} \frac{\xi}{|\xi|}-\eta+(|\eta|-1)_{+} \frac{\eta}{|\eta|}\right\rangle .
$$

Using the fact that $|\xi|,|\eta|>1$ and the Cauchy-Schwarz inequality, we get

$$
\begin{aligned}
B & =\left\langle H_{q-1}(\xi)-H_{q-1}(\eta), \frac{\xi}{|\xi|}-\frac{\eta}{|\eta|}\right\rangle \\
& =(|\xi|-1)^{q-1}+(|\eta|-1)^{q-1}-\frac{\langle\xi, \eta\rangle}{|\xi||\eta|}\left[(|\xi|-1)^{q-1}+(|\eta|-1)^{q-1}\right] \\
& \geq(|\xi|-1)^{q-1}+(|\eta|-1)^{q-1}-\frac{|\xi||\eta|}{|\xi||\eta|}\left[(|\xi|-1)^{q-1}+(|\eta|-1)^{q-1}\right]=0,
\end{aligned}
$$

from which the assertion directly follows.

Finally, let $|\xi|>1$ and $|\eta| \leq 1$. In this case, we have $H_{q-1}(\eta)=H_{\frac{q}{2}}(\eta)=0$ and we can write the left-hand side of (2.7) as the difference of two terms $D-E$, where

$$
D:=\left\langle H_{q-1}(\xi), \xi-\eta\right\rangle \quad \text { and } \quad E:=\left\langle H_{q-1}(\xi), \frac{\xi}{|\xi|}-\eta\right\rangle
$$

Using the fact that $|\xi|>1,|\eta| \leq 1$ and the Cauchy-Schwarz inequality, we find that

$$
E=(|\xi|-1)^{q-1}-\frac{\langle\xi, \eta\rangle}{|\xi|}(|\xi|-1)^{q-1} \geq(|\xi|-1)^{q-1}-\frac{|\xi||\eta|}{|\xi|}(|\xi|-1)^{q-1} \geq 0,
$$

which concludes the proof. 


\subsection{Besov-Lipschitz spaces}

Given $h \in \mathbb{R}^{n}$ and $v: \mathbb{R}^{n} \rightarrow \mathbb{R}$, let us introduce the notations $\tau_{h} v(x)=v(x+h)$ and $\Delta_{h}[v](x)=v(x+h)-v(x)$. As in [27, Section 2.5.12], given $0<\alpha<1$ and $1 \leq p, q<\infty$, we say that $v$ belongs to the Besov space $B_{p, q}^{\alpha}\left(\mathbb{R}^{n}\right)$ if $v \in L^{p}\left(\mathbb{R}^{n}\right)$ and

$$
[v]_{\dot{B}_{p, q}^{\alpha}\left(\mathbb{R}^{n}\right)}:=\left(\int_{\mathbb{R}^{n}}\left(\int_{\mathbb{R}^{n}} \frac{\left|\Delta_{h}[v](x)\right|^{p}}{|h|^{\alpha p}} d x\right)^{\frac{q}{p}} \frac{d h}{|h|^{n}}\right)^{\frac{1}{q}}<\infty .
$$

One can define a norm on the space $B_{p, q}^{\alpha}\left(\mathbb{R}^{n}\right)$ as follows

$$
\|v\|_{B_{p, q}^{\alpha}\left(\mathbb{R}^{n}\right)}:=\|v\|_{L^{p}\left(\mathbb{R}^{n}\right)}+[v]_{\dot{B}_{p, q}^{\alpha}\left(\mathbb{R}^{n}\right)},
$$

and with this norm $B_{p, q}^{\alpha}\left(\mathbb{R}^{n}\right)$ is a Banach space. Equivalently, we could simply say that a function $v \in L^{p}\left(\mathbb{R}^{n}\right)$ belongs to $B_{p, q}^{\alpha}\left(\mathbb{R}^{n}\right)$ if and only if $\frac{\Delta_{h}[v]}{|h|^{\alpha}} \in L^{q}\left(\frac{d h}{|h|^{n}} ; L^{p}\left(\mathbb{R}^{n}\right)\right)$. As usual, in (2.8) if one simply integrates for $h \in B(0, \delta)$ for a fixed $\delta>0$, then an equivalent norm is obtained, since

$$
\left(\int_{\{|h| \geq \delta\}}\left(\int_{\mathbb{R}^{n}} \frac{\left|\Delta_{h}[v](x)\right|^{p}}{|h|^{\alpha p}} d x\right)^{\frac{q}{p}} \frac{d h}{|h|^{n}}\right)^{\frac{1}{q}} \leq c(n, \alpha, p, q, \delta)\|v\|_{L^{p}\left(\mathbb{R}^{n}\right)}
$$

Similarly, for a function $v \in L^{p}\left(\mathbb{R}^{n}\right)$ we say that $v \in B_{p, \infty}^{\alpha}\left(\mathbb{R}^{n}\right)$ if

$$
[v]_{\dot{B}_{p, \infty}^{\alpha}\left(\mathbb{R}^{n}\right)}:=\sup _{h \in \mathbb{R}^{n}}\left(\int_{\mathbb{R}^{n}} \frac{\left|\Delta_{h}[v](x)\right|^{p}}{|h|^{\alpha p}} d x\right)^{\frac{1}{p}}<\infty,
$$

and we can define the following norm

$$
\|v\|_{B_{p, \infty}^{\alpha}\left(\mathbb{R}^{n}\right)}:=\|v\|_{L^{p}\left(\mathbb{R}^{n}\right)}+[v]_{\dot{B}_{p, \infty}^{\alpha}\left(\mathbb{R}^{n}\right)} .
$$

Again, in (2.9) one can simply take the supremum over $|h| \leq \delta$ for a fixed $\delta>0$, thus obtaining an equivalent norm. By construction, $B_{p, q}^{\alpha}\left(\mathbb{R}^{n}\right) \subset L^{p}\left(\mathbb{R}^{n}\right)$. Moreover, one also has the following version of Sobolev embeddings (a proof can be found in [21, Proposition 7.12], taking into account that $L^{r}=F_{r, 2}^{0}$, with $\left.1<r<+\infty\right)$ :

Lemma 2.6. Suppose that $0<\alpha<1$.

(a) If $1<p<\frac{n}{\alpha}$ and $1 \leq q \leq p_{\alpha}^{*}:=\frac{n p}{n-\alpha p}$, then there exists a continuous embedding $B_{p, q}^{\alpha}\left(\mathbb{R}^{n}\right) \hookrightarrow$ $L^{p_{\alpha}^{*}}\left(\mathbb{R}^{n}\right)$.

(b) If $p=\frac{n}{\alpha}$ and $1 \leq q \leq \infty$, then there exists a continuous embedding $B_{p, q}^{\alpha}\left(\mathbb{R}^{n}\right) \hookrightarrow B M O\left(\mathbb{R}^{n}\right)$, where BMO denotes the space of functions with bounded mean oscillations [20, Chapter 2].

For further needs, we recall the following inclusions (see [21, Proposition 7.10 and Formula $(7.35)])$.

Lemma 2.7. Suppose that $0<\beta<\alpha<1$. 
(a) If $1<p \leq+\infty$ and $1 \leq q \leq r \leq+\infty$ then $B_{p, q}^{\alpha}\left(\mathbb{R}^{n}\right) \subset B_{p, r}^{\alpha}\left(\mathbb{R}^{n}\right)$.

(b) If $1<p \leq+\infty$ and $1 \leq q, r \leq+\infty$ then $B_{p, q}^{\alpha}\left(\mathbb{R}^{n}\right) \subset B_{p, r}^{\beta}\left(\mathbb{R}^{n}\right)$.

(c) If $1 \leq q \leq+\infty$, then $B_{\frac{n}{\alpha}, q}^{\alpha}\left(\mathbb{R}^{n}\right) \subset B_{\frac{n}{\beta}, q}^{\beta}\left(\mathbb{R}^{n}\right)$.

Combining Lemmas 2.6 and 2.7. we obtain the following Sobolev-type embedding theorem for Besov spaces $B_{p, \infty}^{\alpha}\left(\mathbb{R}^{n}\right)$ that are excluded from assumptions (a) in Lemma 2.6.

Theorem 2.8. Suppose that $0<\beta<\alpha<1$. If $1<p<\frac{n}{\alpha}$, then there exists a continuous embedding $B_{p, \infty}^{\alpha}\left(\mathbb{R}^{n}\right) \hookrightarrow L^{p_{\beta}^{*}}\left(\mathbb{R}^{n}\right)$. Moreover, for every $v \in B_{p, \infty}^{\alpha}\left(\mathbb{R}^{n}\right)$, the following local estimate

$$
\|v\|_{L^{\frac{n p}{n-\beta p}\left(B_{\rho}\right)}} \leq c\left([v]_{\dot{B}_{p, \infty}^{\alpha}\left(B_{R}\right)}+\|v\|_{L^{p}\left(B_{R}\right)}\right)
$$

holds for every ball $B_{\rho} \subset B_{R}$ with $c=c(n, R, \rho, \alpha, \beta)$.

We can also define local Besov spaces as follows. Given a domain $\Omega \subset \mathbb{R}^{n}$, we say that a function $v$ belongs to $B_{p, q, l o c}^{\alpha}(\Omega)$ if $\varphi v \in B_{p, q}^{\alpha}\left(\mathbb{R}^{n}\right)$ whenever $\varphi$ belongs to the class $C_{c}^{\infty}(\Omega)$ of smooth functions with compact support contained in $\Omega$. It is worth noticing that one can prove suitable versions of Lemmas 2.6 and 2.7, by using local Besov spaces.

The following lemma is an easy consequence of the definitions given above and its proof can be found in [3].

Lemma 2.9. A function $v \in L_{l o c}^{p}(\Omega)$ belongs to the local Besov space $B_{p, q, l o c}^{\alpha}(\Omega)$ if and only if

$$
\left\|\frac{\Delta_{h}[v]}{|h|^{\alpha}}\right\|_{L^{q}\left(\frac{d h}{\left.|h|^{n} ; L^{p}(B)\right)}\right.}<\infty
$$

for any ball $B \subset B_{2 r_{B}} \Subset \Omega$ with radius $r_{B}$. Here, the balls $B$ and $B_{2 r_{B}}$ are supposed to be concentric and the measure $\frac{d h}{|h|^{n}}$ is restricted to the ball $B\left(0, r_{B}\right)$ on the $h$-space.

Finally, the following result represents, in some sense, the local counterpart of Lemma 2.6 in the case $q=\infty$ :

Theorem 2.10. On any domain $\Omega \subset \mathbb{R}^{n}$ we have the continuous embedding $B_{p, \infty, l o c}^{\alpha}(\Omega) \hookrightarrow$ $L_{l o c}^{r}(\Omega)$ for all $r<\frac{n p}{n-\alpha p}$, provided $\alpha \in(0,1)$ and $1<p<\frac{n}{\alpha}$.

We refer to [25, Sections 30-32] for a proof of this theorem. In fact, the above statement follows by localizing the corresponding result proved for functions defined on $\mathbb{R}^{n}$ in [25], by simply using a smooth cut off function.

\subsection{Difference quotients}

We recall here the definition and some elementary properties of the difference quotients that will be useful in the following (see, for example, [20]).

Definition 2.11. Given $h \in \mathbb{R} \backslash\{0\}$, for every vector-valued function $F: \Omega \rightarrow \mathbb{R}^{N}$ defined in an open set $\Omega \subset \mathbb{R}^{n}$, we call the difference quotient of $F$ with respect to $x_{s}$ the function

$$
\Delta_{s, h} F(x)=\frac{\tau_{s, h} F(x)-F(x)}{h}=\frac{F\left(x+h e_{s}\right)-F(x)}{h},
$$


where $e_{s}$ is the unit vector in the $x_{s}$ direction and $s \in\{1, \ldots, n\}$.

When no confusion can arise, we shall omit the index $s$ and simply write $\Delta_{h}$ or $\tau_{h}$ instead of $\Delta_{s, h}$ or $\tau_{s, h}$, respectively.

Proposition 2.12. Let $F$ and $G$ be two functions such that $F, G \in W^{1, p}(\Omega)$, with $p \geq 1$, and let us consider the set

$$
\Omega_{|h|}:=\{x \in \Omega: \operatorname{dist}(x, \partial \Omega)>|h|\} .
$$

Then:

(a) $\Delta_{h} F \in W^{1, p}\left(\Omega_{|h|}\right)$ and

$$
D_{i}\left(\Delta_{h} F\right)=\Delta_{h}\left(D_{i} F\right), \quad \text { for every } i \in\{1, \ldots, n\} .
$$

(b) If at least one of the functions $F$ or $G$ has support contained in $\Omega_{|h|}$, then

$$
\int_{\Omega} F \Delta_{h} G d x=-\int_{\Omega} G \Delta_{-h} F d x
$$

(c) We have

$$
\Delta_{h}(F G)(x)=F\left(x+h e_{s}\right) \Delta_{h} G(x)+G(x) \Delta_{h} F(x) .
$$

The next result is a kind of integral version of Lagrange Theorem and it will be used to prove Theorems 1.1 and 4.1 .

Lemma 2.13. If $0<\rho<R$, $|h|<\frac{R-\rho}{2}, 1<p<+\infty$, and $F \in L^{p}\left(B_{R}, \mathbb{R}^{N}\right)$, DF $\in$ $L^{p}\left(B_{R}, \mathbb{R}^{N \times n}\right)$, then

$$
\int_{B_{\rho}}\left|F\left(x+h e_{s}\right)-F(x)\right|^{p} d x \leq c^{p}(n)|h|^{p} \int_{B_{R}}|D F(x)|^{p} d x,
$$

where the balls $B_{\rho}$ and $B_{R}$ are supposed to be concentric. Moreover

$$
\int_{B_{\rho}}\left|F\left(x+h e_{s}\right)\right|^{p} d x \leq \int_{B_{R}}|F(x)|^{p} d x .
$$

\section{The singular case $1<q<2$}

Having declared our aims and introduced all the required tools, we now come to the

Proof of Theorem 1.1. We first observe that $H_{\frac{q}{2}}(\nabla u) \in L_{l o c}^{2}(\Omega)$ and $H_{q-1}(\nabla u) \in L_{l o c}^{p}(\Omega)$, where $p=q^{\prime}=q /(q-1)$. Let $e_{s}$ be a coordinate direction in $\mathbb{R}^{n}$; from now on, by a slight abuse of notation, we shall write $\tau_{h} g(x)$ to denote $g\left(x+h e_{s}\right)$, where $h \in \mathbb{R} \backslash\{0\}$.

Now, let $\varphi \in W^{1, q}(\Omega)$ be compactly supported in $\Omega$ (i.e. a test function) and $h \in \mathbb{R} \backslash\{0\}$ be such that $|h|<\operatorname{dist}\left(\operatorname{supp}(\varphi), \mathbb{R}^{n} \backslash \Omega\right)$. Since $u$ is a local weak solution of equation (1.6), we have

$$
\int_{\Omega}\left\langle\Delta_{h} H_{q-1}(\nabla u), \nabla \varphi\right\rangle d x=\int_{\Omega}\left(\Delta_{h} f\right) \varphi d x .
$$


Let us fix two concentric balls $B_{R / 2}$ and $B_{R}$ with $B_{R} \Subset \Omega$, and consider a cut off function $\xi \in C_{c}^{\infty}\left(B_{R}\right)$ such that $\xi \equiv 1$ on $\bar{B}_{R / 2}$ and $\|\nabla \xi\|_{\infty} \leq C / R$. Let $h \in \mathbb{R} \backslash\{0\}$ be such that $|h| \leq r_{0}<\frac{1}{2} \operatorname{dist}\left(B_{R}, \mathbb{R}^{n} \backslash \Omega\right)$. In what follows, we will denote by $c_{k}$ and $c$ some positive constants which do not depend on $h$, but they may vary on different occasions.

Now, let us consider, first, the case $f \in B_{p, \infty, l o c}^{\alpha}(\Omega)$. We then choose $\varphi=\xi^{2} \Delta_{h} u$ as a test function into (3.1). Using the fact that $u \in W_{l o c}^{1, q}(\Omega), f \in B_{p, \infty, l o c}^{\alpha}(\Omega)$, Hölder's inequality and the properties of the difference quotients, and defining $B^{\prime}:=B_{R}+B\left(0, r_{0}\right)$, we get

$$
\begin{aligned}
& \int_{\Omega}\left\langle\Delta_{h} H_{q-1}(\nabla u), \xi^{2} \Delta_{h} \nabla u+2 \xi \nabla \xi \Delta_{h} u\right\rangle d x=\int_{\Omega}\left\langle\Delta_{h} H_{q-1}(\nabla u), \nabla \varphi\right\rangle d x \\
& =\int_{B_{R}}\left(\Delta_{h} f\right) \varphi d x \leq \int_{B_{R}}\left|\Delta_{h} f\right| \xi^{2}\left|\Delta_{h} u\right| d x \leq\left(\int_{B_{R}}\left|\Delta_{h} f\right|^{p} d x\right)^{\frac{1}{p}}\left(\int_{B_{R}} \xi^{2 q}\left|\Delta_{h} u\right|^{q} d x\right)^{\frac{1}{q}} \\
& \leq\left(\int_{B_{R}} \frac{\left|\Delta_{h} f\right|^{p}}{|h|^{\alpha p}} d x\right)^{\frac{1}{p}}|h|^{\alpha}\left\|\Delta_{h} u\right\|_{L^{q}\left(B_{R}\right)} \leq c_{1}(n)\left(\int_{B_{R}} \frac{\left|\tau_{h} f-f\right|^{p}}{|h|^{\alpha p}} d x\right)^{\frac{1}{p}}|h|^{\alpha-1}\|\nabla u\|_{L^{q}\left(B^{\prime}\right)} \\
& \leq c_{1}(n)\|f\|_{B_{p, \infty}^{\alpha}\left(B^{\prime}\right)}\|\nabla u\|_{L^{q}\left(B^{\prime}\right)}|h|^{\alpha-1} .
\end{aligned}
$$

From the previous estimate, we then obtain

$$
\begin{aligned}
& \int_{\Omega} \xi^{2}\left\langle\Delta_{h} H_{q-1}(\nabla u), \Delta_{h} \nabla u\right\rangle d x \\
& \leq c_{1}(n)\|f\|_{B_{p, \infty}^{\alpha}\left(B^{\prime}\right)}\|\nabla u\|_{L^{q}\left(B^{\prime}\right)}|h|^{\alpha-1}-2 \int_{\Omega}\left\langle\Delta_{h} H_{q-1}(\nabla u), \nabla \xi\right\rangle \xi \Delta_{h} u d x .
\end{aligned}
$$

Now, by Lemma 2.5 we have

$$
\beta(q, n) \int_{B_{R}} \xi^{2}\left|\Delta_{h} H_{\frac{q}{2}}(\nabla u(x))\right|^{2} d x \leq \int_{\Omega} \xi^{2}\left\langle\Delta_{h} H_{q-1}(\nabla u(x)), \Delta_{h} \nabla u(x)\right\rangle d x
$$

where $\beta(q, n)$ is a positive constant. Combining estimates (3.2) and (3.3) and applying the Cauchy-Schwarz inequality as well as the properties of $\xi$, we get

$$
\begin{aligned}
& \beta(q, n) \int_{B_{R}} \xi^{2}\left|\Delta_{h} H_{\frac{q}{2}}(\nabla u)\right|^{2} d x \\
& \leq c_{1}(n)\|f\|_{B_{p, \infty}^{\alpha}\left(B^{\prime}\right)}\|\nabla u\|_{L^{q}\left(B^{\prime}\right)}|h|^{\alpha-1}-2 \int_{\Omega}\left\langle\Delta_{h} H_{q-1}(\nabla u), \nabla \xi\right\rangle \xi \Delta_{h} u d x \\
& \leq c_{1}(n)\|f\|_{B_{p, \infty}^{\alpha}\left(B^{\prime}\right)}\|\nabla u\|_{L^{q}\left(B^{\prime}\right)}|h|^{\alpha-1}+c_{2}(R) \int_{\Omega}\left|\Delta_{h} H_{q-1}(\nabla u)\right| \xi\left|\Delta_{h} u\right| d x,
\end{aligned}
$$

and dividing by $\beta(q, n)$, we obtain

$$
\begin{aligned}
& \int_{B_{R}} \xi^{2}\left|\Delta_{h} H_{\frac{q}{2}}(\nabla u)\right|^{2} d x \\
& \leq c_{3}(q, n)\|f\|_{B_{p, \infty}^{\alpha}\left(B^{\prime}\right)}\|\nabla u\|_{L^{q}\left(B^{\prime}\right)}|h|^{\alpha-1}+c_{4}(R, q, n) \int_{\Omega}\left|\Delta_{h} H_{q-1}(\nabla u)\right| \xi\left|\Delta_{h} u\right| d x .
\end{aligned}
$$


Now we set

$$
I_{1}:=\int_{\Omega}\left|\Delta_{h} H_{q-1}(\nabla u)\right| \xi\left|\Delta_{h} u\right| d x
$$

and apply Hölder's inequality with exponents $(q, p)$ and the properties of the difference quotients to estimate $I_{1}$ as follows:

$$
I_{1} \leq c_{1}(n)\|\nabla u\|_{L^{q}\left(B^{\prime}\right)}\left(\int_{B_{R}} \xi^{p}\left|\Delta_{h} H_{q-1}(\nabla u)\right|^{p} d x\right)^{\frac{1}{p}} .
$$

Similarly, we set

$$
I_{2}:=\int_{B_{R}} \xi^{p}\left|\Delta_{h} H_{q-1}(\nabla u)\right|^{p} d x
$$

and use Lemma 2.3. Hölder's inequality with exponents $\left(\frac{2}{q}, \frac{2}{2-q}\right)$ and the properties of the difference quotients to control $I_{2}$ as follows:

$$
\begin{gathered}
I_{2} \leq c_{5}(q, n) \int_{B_{R}} \xi^{p}\left|\Delta_{h} H_{1}(\nabla u)\right|^{p}\left[\left(\left|\tau_{h} \nabla u\right|-1\right)_{+}+(|\nabla u|-1)_{+}\right]^{p(q-2)} d x \\
=c_{5}(q, n) \int_{B_{R}} \xi^{p}\left|\Delta_{h} H_{1}(\nabla u)\right|^{q}\left|\Delta_{h} H_{1}(\nabla u)\right|^{p-q}\left[\left(\left|\tau_{h} \nabla u\right|-1\right)_{+}+(|\nabla u|-1)_{+}\right]^{p(q-2)} d x \\
=c_{5}(q, n)|h|^{q-p} \int_{B_{R}} \xi^{p}\left|\Delta_{h} H_{1}(\nabla u)\right|^{q}\left|H_{1}\left(\tau_{h} \nabla u\right)-H_{1}(\nabla u)\right|^{p-q}\left[\left(\left|\tau_{h} \nabla u\right|-1\right)_{+}+(|\nabla u|-1)_{+}\right]^{p(q-2)} d x \\
\leq c_{5}(q, n)|h|^{q-p} \int_{B_{R}} \xi^{p}\left|\Delta_{h} H_{1}(\nabla u)\right|^{q}\left[\left(\left|\tau_{h} \nabla u\right|-1\right)_{+}+(|\nabla u|-1)_{+}\right]^{p q-(p+q)} d x \\
=c_{5}(q, n)|h|^{q-p} \int_{B_{R}} \xi^{p}\left|\Delta_{h} H_{1}(\nabla u)\right|^{q}\left[\left(\left|\tau_{h} \nabla u\right|-1\right)_{+}+(|\nabla u|-1)_{+}\right]^{\frac{q}{2}(q-2)+\frac{q}{2}(2-q)} d x \\
\leq c_{5}(q, n)|h|^{q-p}\left(\int_{B_{R}} \xi^{\frac{2}{q-1}}\left|\Delta_{h} H_{1}(\nabla u)\right|^{2}\left[\left(\left|\tau_{h} \nabla u\right|-1\right)_{+}+(|\nabla u|-1)_{+}\right]^{q-2} d x\right)^{\frac{q}{2}} 2^{\frac{q(2-q)}{2}}\|\nabla u\|_{L^{q\left(B^{\prime}\right)}}^{\frac{q(2-q)}{2}} \\
\leq c_{6}(q, n)|| \nabla u \|_{L^{\left(q\left(B^{\prime}\right)\right.}}^{\frac{q(2-q)}{2}}|h|^{q-p}\left(\int_{B_{R}} \xi^{2}\left|\Delta_{h} H_{\frac{q}{2}}(\nabla u)\right|^{2} d x\right)^{\frac{q}{2}},
\end{gathered}
$$

where, in the last line, we have used Lemma 2.3 again, as well as the properties of $\xi$. Collecting estimates (3.4), (3.5) and (3.6) and applying Young's inequality with $\theta>0$ and exponents $\left(\frac{2}{q-1}, \frac{2}{3-q}\right)$, we obtain

$$
\begin{aligned}
& \int_{B_{R}} \xi^{2}\left|\Delta_{h} H_{\frac{q}{2}}(\nabla u)\right|^{2} d x \leq c_{3}(q, n)\|f\|_{B_{p, \infty}^{\alpha}\left(B^{\prime}\right)}\|\nabla u\|_{L^{q}\left(B^{\prime}\right)}|h|^{\alpha-1}+c_{7}(R, q, n)\|\nabla u\|_{L^{q}\left(B^{\prime}\right)} I_{2}^{1 / p} \\
& \leq c_{3}(q, n)\|f\|_{B_{p, \infty}^{\alpha}\left(B^{\prime}\right)}\|\nabla u\|_{L^{q}\left(B^{\prime}\right)}|h|^{\alpha-1}+c_{8}(R, q, n)\|\nabla u\|_{L^{q}\left(B^{\prime}\right)}^{\frac{q(3-q)}{2}}|h|^{q-2}\left(\int_{B_{R}} \xi^{2}\left|\Delta_{h} H_{\frac{q}{2}}(\nabla u)\right|^{2} d x\right)^{\frac{q-1}{2}} \\
& \leq c_{3}(q, n)\|f\|_{B_{p, \infty}^{\alpha}\left(B^{\prime}\right)}\|\nabla u\|_{L^{q}\left(B^{\prime}\right)}|h|^{\alpha-1} \\
& \quad+\frac{q-1}{2} \theta^{\frac{1}{q-1}} \int_{B_{R}} \xi^{2}\left|\Delta_{h} H_{\frac{q}{2}}(\nabla u)\right|^{2} d x+\frac{3-q}{2} \theta^{\frac{1}{q-3}} c_{9}(R, q, n)\|\nabla u\|_{L^{q}\left(B^{\prime}\right)}^{q}|h|^{\frac{2(q-2)}{3-q}} .
\end{aligned}
$$


Choosing $\theta=\left(\frac{1}{q-1}\right)^{q-1}$ and reabsorbing the integral in the right-hand side of (3.7) by the left-hand side, we get

$$
\int_{B_{R}} \xi^{2}\left|\Delta_{h} H_{\frac{q}{2}}(\nabla u)\right|^{2} d x \leq c\|f\|_{B_{p, \infty}^{\alpha}\left(B^{\prime}\right)}\|\nabla u\|_{L^{q}\left(B^{\prime}\right)}|h|^{\alpha-1}+c\|\nabla u\|_{L^{q}\left(B^{\prime}\right)}^{q}|h|^{\frac{2(q-2)}{3-q}}
$$

from which we can infer

$$
\int_{B_{R / 2}}\left|\Delta_{h} H_{\frac{q}{2}}(\nabla u)\right|^{2} d x \leq c\|f\|_{B_{p, \infty}^{\alpha}\left(B^{\prime}\right)}\|\nabla u\|_{L^{q}\left(B^{\prime}\right)}|h|^{\alpha-1}+c\|\nabla u\|_{L^{q}\left(B^{\prime}\right)}^{q}|h|^{\frac{2(q-2)}{3-q}}
$$

with $c=c(R, q, n)>0$. Now, let $\lambda=\min \left\{\frac{\alpha+1}{2}, \frac{1}{3-q}\right\}$. Dividing both sides of (3.8) by $|h|^{2 \lambda-2}$, we then have

$$
\int_{B_{R / 2}}\left|\frac{\tau_{h} H_{q / 2}(\nabla u)-H_{q / 2}(\nabla u)}{|h|^{\lambda}}\right|^{2} d x \leq c\left(\|f\|_{B_{p, \infty}^{\alpha}\left(B^{\prime}\right)}\|\nabla u\|_{L^{q}\left(B^{\prime}\right)}|h|^{\alpha+1-2 \lambda}+\|\nabla u\|_{L^{q}\left(B^{\prime}\right)}^{q}|h|^{\frac{2}{3-q}-2 \lambda}\right) .
$$

Since the above estimate holds for every $h \in \mathbb{R} \backslash\{0\}$ such that $|h| \leq r_{0}$, we can take the supremum over $|h|<\delta$ for some $\delta<r_{0}$ and obtain

$$
\sup _{|h|<\delta} \int_{B_{R / 2}}\left|\frac{\tau_{h} H_{q / 2}(\nabla u)-H_{q / 2}(\nabla u)}{\mid h^{\lambda}}\right|^{2} d x \leq c\left(\|f\|_{B_{p, \infty}^{\alpha}\left(B^{\prime}\right)}\|\nabla u\|_{L^{q}\left(B^{\prime}\right)} r_{0}^{\alpha+1-2 \lambda}+\|\nabla u\|_{L^{q}\left(B^{\prime}\right)}^{q} r_{0}^{\frac{2}{3-q}-2 \lambda}\right) .
$$

In particular, this means that $H_{\frac{q}{2}}(\nabla u) \in B_{2, \infty}^{\min \left\{\frac{\alpha+1}{2}, \frac{1}{3-q}\right\}}$ locally in $\Omega$.

Finally, let us consider the case $f \in W_{l o c}^{1, p}(\Omega)$. Arguing as above, but this time using Lemma 2.13 to estimate the $L^{p}$-norm of the difference quotient of $f$, we obtain

$$
\int_{B_{R / 2}}\left|\frac{\tau_{h} H_{q / 2}(\nabla u)-H_{q / 2}(\nabla u)}{|h|^{1 /(3-q)}}\right|^{2} d x \leq c\left(\|\nabla f\|_{L^{p}\left(B^{\prime}\right)}\|\nabla u\|_{L^{q}\left(B^{\prime}\right)}|h|^{\frac{2(2-q)}{3-q}}+\|\nabla u\|_{L^{q}\left(B^{\prime}\right)}^{q}\right),
$$

which holds for every $h \in \mathbb{R} \backslash\{0\}$ such that $|h| \leq r_{0}$. Arguing exactly as before, we may then conclude that $H_{\frac{q}{2}}(\nabla u) \in B_{2, \infty, l o c}^{\frac{1}{3-q}}(\Omega)$.

We are now in position to give the

Proof of Theorem 1.2. Let us begin with the case $f \in W_{l o c}^{1, p}(\Omega)$. By duality, we know that $\sigma_{0}$ is related to any solution $u$ of problem ( $(\mathrm{P} 2)$ through the optimality condition

$$
\sigma_{0}(x)=\nabla \mathcal{H}^{*}(\nabla u(x))=H_{q-1}(\nabla u(x)), \text { for } \mathscr{L}^{n} \text {-a.e. } x \in \Omega \text {. }
$$

Now we observe that for every $q \in(1,2)$ we have

$$
0<q-1<\frac{q}{2}
$$


If $B_{R / 2}$ is the same ball fixed in the proof of Theorem 1.1, then applying Lemma 2.3 we get

$$
\begin{aligned}
& \int_{B_{R / 2}}\left|\frac{\tau_{h} \sigma_{0}-\sigma_{0}}{|h|^{\frac{2}{p(3-q)}}}\right|^{p} d x \leq c(q, n)|h|^{\frac{2}{q-3}} \int_{B_{R / 2}}\left|\tau_{h} H_{\frac{q}{2}}(\nabla u)-H_{\frac{q}{2}}(\nabla u)\right|^{p}\left[\left(\left|\tau_{h} \nabla u\right|-1\right)_{+}^{\frac{q}{2}}+(|\nabla u|-1)_{+}^{\frac{q}{2}}\right]^{\frac{q-2}{q-1}} d x \\
& =c(q, n) \int_{B_{R / 2}}\left|\frac{\tau_{h} H_{q / 2}(\nabla u)-H_{q / 2}(\nabla u)}{|h|^{1 /(3-q)}}\right|^{2}\left|\tau_{h} H_{\frac{q}{2}}(\nabla u)-H_{\frac{q}{2}}(\nabla u)\right|^{p-2}\left[\left(\left|\tau_{h} \nabla u\right|-1\right)_{+}^{\frac{q}{2}}+(|\nabla u|-1)_{+}^{\frac{q}{2}}\right]^{\frac{q-2}{q-1}} d x \\
& \leq c(q, n) \int_{B_{R / 2}}\left|\frac{\tau_{h} H_{q / 2}(\nabla u)-H_{q / 2}(\nabla u)}{|h|^{1 /(3-q)}}\right|^{2} d x .
\end{aligned}
$$

Combining estimates (3.11) and (3.10) and arguing as in the last part of the proof of Theorem 1.1, we obtain the desired conclusion.

Finally, when $f \in B_{p, \infty, l o c}^{\alpha}(\Omega)$, a similar argument applies, but taking into account estimate (3.9) instead of (3.10).

Now we observe that the Besov regularity of $\sigma_{0}$ established in Theorem 1.2 allows us to get a gain of integrability for $\sigma_{0}$. More precisely, we have:

Proof of Corollary 1.4. We first note that for every $q \in(1,2)$ and $\alpha \in(0,1)$ we have

$$
\frac{2}{3-q}<2 \leq n \quad \text { and } \quad \alpha+1<n
$$

If $f \in B_{p, \infty, l o c}^{\alpha}(\Omega)$ and $\frac{\alpha+1}{p} \geq \frac{2}{p(3-q)}$ (that is, $\frac{q-1}{3-q} \leq \alpha<1$ ), then from Theorems 1.2 and 2.10 it follows that $\sigma_{0} \in L_{l o c}^{s}(\Omega)$ for all $s \in\left[1, \frac{n p(3-q)}{n(3-q)-2}\right)$.

When $f \in W_{l o c}^{1, p}(\Omega)$, thanks to Theorem 1.2 we have that $\sigma_{0} \in B_{p, \infty, l o c}^{\frac{2}{p(3-q)}}(\Omega)$. We therefore reach the same conclusion as in the previous case.

Finally, when $f \in B_{p, \infty, l o c}^{\alpha}(\Omega)$ and $\frac{\alpha+1}{p}<\frac{2}{p(3-q)}$ (that is, $0<\alpha<\frac{q-1}{3-q}$ ), Theorems 1.2 and 2.10 imply that $\sigma_{0} \in L_{l o c}^{s}(\Omega)$ for all $s \in\left[1, \frac{n p}{n-\alpha-1}\right)$.

As an easy consequence of the (local) higher integrability of $H_{q-1}(\nabla u)$ established in the proof of Corollary 1.4, we get a gain of integrability for $\nabla u$. More precisely, we have:

Proof of Corollary 1.5. In the case $f \in W_{l o c}^{1, p}(\Omega)$, we get $H_{q-1}(\nabla u) \in L_{l o c}^{s}(\Omega)$ for every $s \in\left[1, \frac{n p(3-q)}{n(3-q)-2}\right)$ and then

$$
\int_{K}(|\nabla u(x)|-1)_{+}^{(q-1) s} d x=\int_{K}\left|H_{q-1}(\nabla u(x))\right|^{s} d x<+\infty
$$

for all $s \in\left[1, \frac{n p(3-q)}{n(3-q)-2}\right)$ and all compact subsets $K$ of $\Omega$, which ensures that $\nabla u \in L_{l o c}^{r}(\Omega)$ for all $r \in\left[1, \frac{n q(3-q)}{n(3-q)-2}\right)$. In the other two cases, a similar argument applies, thus proving the assertion. 


\section{The non-singular case $q \geq 2$}

Here we proceed with the analysis of the non-singular case, by proving that, unlike what may occur in the singular one, the Besov regularity of the datum $f$ translates into a Besov regularity for $H_{\frac{q}{2}}(\nabla u)$ with no loss in the order of differentiation. More precisely, we have the following

Theorem 4.1. Let $n \geq 2, q \geq 2, \alpha \in(0,1)$ and $f \in B_{p, \infty, l o c}^{\alpha}(\Omega)$. Moreover, let $u \in W_{\text {loc }}^{1, q}(\Omega)$ be a local weak solution of the equation

$$
-\operatorname{div}\left(\nabla \mathcal{H}^{*}(\nabla u)\right)=f
$$

Then $H_{\frac{q}{2}}(\nabla u) \in B_{2, \infty, l o c}^{\frac{\alpha+1}{2}}(\Omega)$. Furthermore, for any ball $B_{R} \Subset \Omega$, the following estimate

$$
\int_{B_{R / 2}}\left|\tau_{s, h} H_{\frac{q}{2}}(\nabla u)-H_{\frac{q}{2}}(\nabla u)\right|^{2} d x \leq c\left(\|f\|_{B_{p, \infty}^{\alpha}\left(B^{\prime}\right)}\|\nabla u\|_{L^{q}\left(B^{\prime}\right)}|h|^{\alpha+1}+\|\nabla u\|_{L^{q}\left(B^{\prime}\right)}^{q}|h|^{2}\right)
$$

holds true for every $s \in\{1, \ldots, n\}$, for every $h \in \mathbb{R}$ such that $|h| \leq r_{0}<\frac{1}{2} \operatorname{dist}\left(B_{R}, \partial \Omega\right)$, for $B^{\prime}=B_{R}+B\left(0, r_{0}\right)$ and a positive constant $c=c(R, q, n)$.

Proof. We first observe that $H_{\frac{q}{2}}(\nabla u) \in L_{l o c}^{2}(\Omega)$ and $H_{q-1}(\nabla u) \in L_{l o c}^{p}(\Omega)$. Let $e_{s}$ be a coordinate direction in $\mathbb{R}^{n}$; from now on, by a slight abuse of notation, we shall write $\tau_{h} g(x)$ to denote $g\left(x+h e_{s}\right)$, where $h \in \mathbb{R} \backslash\{0\}$.

Now, let $\varphi \in W^{1, q}(\Omega)$ be compactly supported in $\Omega$ (i.e. a test function) and $h \in \mathbb{R} \backslash\{0\}$ be such that $|h|<\operatorname{dist}\left(\operatorname{supp}(\varphi), \mathbb{R}^{n} \backslash \Omega\right)$. Since $u$ is a local weak solution of equation (4.1), we have

$$
\int_{\Omega}\left\langle\Delta_{h} H_{q-1}(\nabla u), \nabla \varphi\right\rangle d x=\int_{\Omega}\left(\Delta_{h} f\right) \varphi d x .
$$

Let us fix two concentric balls $B_{R / 2}$ and $B_{R}$ with $B_{R} \Subset \Omega$, and consider a cut off function $\xi \in C_{c}^{\infty}\left(B_{R}\right)$ such that $\xi \equiv 1$ on $\bar{B}_{R / 2}$ and $\|\nabla \xi\|_{\infty} \leq C / R$. Let $h \in \mathbb{R} \backslash\{0\}$ be such that $|h| \leq$ $r_{0}<\frac{1}{2}$ dist $\left(B_{R}, \mathbb{R}^{n} \backslash \Omega\right)$. In what follows, we will denote by $c_{k}$ and $c$ some positive constants which do not depend on $h$, but they may vary on different occasions. We then choose $\varphi=\xi^{2} \Delta_{h} u$ as a test function into (4.2). Using the fact that $u \in W_{l o c}^{1, q}(\Omega), f \in B_{p, \infty, l o c}^{\alpha}(\Omega)$, Hölder's inequality and the properties of the difference quotients, and defining $B^{\prime}:=B_{R}+B\left(0, r_{0}\right)$, we get

$$
\begin{aligned}
& \int_{\Omega}\left\langle\Delta_{h} H_{q-1}(\nabla u), \xi^{2} \Delta_{h} \nabla u+2 \xi \nabla \xi \Delta_{h} u\right\rangle d x=\int_{\Omega}\left\langle\Delta_{h} H_{q-1}(\nabla u), \nabla \varphi\right\rangle d x \\
& =\int_{B_{R}}\left(\Delta_{h} f\right) \varphi d x \leq \int_{B_{R}}\left|\Delta_{h} f\right| \xi^{2}\left|\Delta_{h} u\right| d x \leq\left(\int_{B_{R}}\left|\Delta_{h} f\right|^{p} d x\right)^{\frac{1}{p}}\left(\int_{B_{R}} \xi^{2 q}\left|\Delta_{h} u\right|^{q} d x\right)^{\frac{1}{q}} \\
& \leq\left(\int_{B_{R}} \frac{\left|\Delta_{h} f\right|^{p}}{|h|^{\alpha p}} d x\right)^{\frac{1}{p}}|h|^{\alpha}\left\|\Delta_{h} u\right\|_{L^{q}\left(B_{R}\right)} \leq c_{1}(n)\left(\int_{B_{R}} \frac{\left|\tau_{h} f-f\right|^{p}}{|h|^{\alpha p}} d x\right)^{\frac{1}{p}}|h|^{\alpha-1}\|\nabla u\|_{L^{q}\left(B^{\prime}\right)} \\
& \leq c_{1}(n)\|f\|_{B_{p, \infty}^{\alpha}\left(B^{\prime}\right)}\|\nabla u\|_{L^{q}\left(B^{\prime}\right)}|h|^{\alpha-1},
\end{aligned}
$$


from which we obtain

$$
\begin{aligned}
& \int_{\Omega} \xi^{2}\left\langle\Delta_{h} H_{q-1}(\nabla u), \Delta_{h} \nabla u\right\rangle d x \\
& \leq c_{1}(n)\|f\|_{B_{p, \infty}^{\alpha}\left(B^{\prime}\right)}\|\nabla u\|_{L^{q}\left(B^{\prime}\right)}|h|^{\alpha-1}-2 \int_{\Omega}\left\langle\Delta_{h} H_{q-1}(\nabla u), \nabla \xi\right\rangle \xi \Delta_{h} u d x .
\end{aligned}
$$

Now, by Lemma 2.4 we have

$$
\frac{4}{q^{2}} \int_{B_{R}} \xi^{2}\left|\Delta_{h} H_{\frac{q}{2}}(\nabla u(x))\right|^{2} d x \leq \int_{\Omega} \xi^{2}\left\langle\Delta_{h} H_{q-1}(\nabla u(x)), \Delta_{h} \nabla u(x)\right\rangle d x .
$$

Using the above estimate together with (4.3) and the Cauchy-Schwarz inequality, we then get

$$
\begin{aligned}
& \frac{4}{q^{2}} \int_{B_{R}} \xi^{2}\left|\Delta_{h} H_{\frac{q}{2}}(\nabla u)\right|^{2} d x \\
& \leq c_{1}(n)\|f\|_{B_{p, \infty}^{\alpha}\left(B^{\prime}\right)}\|\nabla u\|_{L^{q}\left(B^{\prime}\right)}|h|^{\alpha-1}-2 \int_{\Omega}\left\langle\Delta_{h} H_{q-1}(\nabla u), \nabla \xi\right\rangle \xi \Delta_{h} u d x \\
& \leq c_{1}(n)\|f\|_{B_{p, \infty}^{\alpha}\left(B^{\prime}\right)}\|\nabla u\|_{L^{q}\left(B^{\prime}\right)}|h|^{\alpha-1}+2\|\nabla \xi\|_{L^{\infty}} \int_{\Omega}\left|\Delta_{h} H_{q-1}(\nabla u)\right| \xi\left|\Delta_{h} u\right| d x \\
& \leq c_{1}(n)\|f\|_{B_{p, \infty}^{\alpha}\left(B^{\prime}\right)}\|\nabla u\|_{L^{q}\left(B^{\prime}\right)}|h|^{\alpha-1}+c_{2}(R) \int_{\Omega}\left|\Delta_{h} H_{q-1}(\nabla u)\right| \xi\left|\Delta_{h} u\right| d x,
\end{aligned}
$$

and dividing by $4 / q^{2}$, we obtain

$$
\begin{aligned}
& \int_{B_{R}} \xi^{2}\left|\Delta_{h} H_{\frac{q}{2}}(\nabla u)\right|^{2} d x \\
& \leq c_{3}(q, n)\|f\|_{B_{p, \infty}^{\alpha}\left(B^{\prime}\right)}\|\nabla u\|_{L^{q}\left(B^{\prime}\right)}|h|^{\alpha-1}+c_{4}(R, q) \int_{\Omega}\left|\Delta_{h} H_{q-1}(\nabla u)\right| \xi\left|\Delta_{h} u\right| d x .
\end{aligned}
$$

Now, our aim is to estimate

$$
I_{1}:=\int_{\Omega}\left|\Delta_{h} H_{q-1}(\nabla u)\right| \xi\left|\Delta_{h} u\right| d x
$$

By virtue of Lemma 2.4 we have

$$
\left|\Delta_{h} H_{q-1}(\nabla u(x))\right| \leq(q-1)\left(\left|\tau_{h} H_{\frac{q}{2}}(\nabla u(x))\right|^{\frac{q-2}{q}}+\left|H_{\frac{q}{2}}(\nabla u(x))\right|^{\frac{q-2}{q}}\right)\left|\Delta_{h} H_{\frac{q}{2}}(\nabla u(x))\right| .
$$

We now use (4.5), Hölder's inequality with exponents $\left(q, 2, \frac{2 q}{q-2}\right)$ and the properties of the difference quotients to control $I_{1}$ as follows: 


$$
\begin{aligned}
I_{1} \leq & (q-1) \int_{B_{R}} \xi\left|\Delta_{h} u\right|\left(\left|\tau_{h} H_{\frac{q}{2}}(\nabla u)\right|^{\frac{q-2}{q}}+\left|H_{\frac{q}{2}}(\nabla u)\right|^{\frac{q-2}{q}}\right)\left|\Delta_{h} H_{\frac{q}{2}}(\nabla u)\right| d x \\
\leq & (q-1)\left\|\Delta_{h} u\right\|_{L^{q}\left(B_{R}\right)}\left(\int_{B_{R}} \xi^{2}\left|\Delta_{h} H_{\frac{q}{2}}(\nabla u)\right|^{2} d x\right)^{\frac{1}{2}} \\
& \cdot\left(\int_{B_{R}}\left(\left|\tau_{h} H_{\frac{q}{2}}(\nabla u)\right|^{\frac{q-2}{q}}+\left|H_{\frac{q}{2}}(\nabla u)\right|^{\frac{q-2}{q}}\right)^{\frac{2 q}{q-2}} d x\right)^{\frac{q-2}{2 q}} \\
\leq & c_{5}(q, n)\|\nabla u\|_{L^{q}\left(B^{\prime}\right)}\left(\int_{B_{R}} \xi^{2}\left|\Delta_{h} H_{\frac{q}{2}}(\nabla u)\right|^{2} d x\right)^{\frac{1}{2}} 2\left\|H_{\frac{q}{2}}(\nabla u)\right\|_{L^{2}\left(B^{\prime}\right)}^{\frac{q-2}{q}} \\
\leq & c_{6}(q, n)\|\nabla u\|_{L^{q}\left(B^{\prime}\right)}^{q / 2}\left(\int_{B_{R}} \xi^{2}\left|\Delta_{h} H_{\frac{q}{2}}(\nabla u)\right|^{2} d x\right)^{\frac{1}{2}} \cdot
\end{aligned}
$$

Joining (4.4) and (4.6) and applying Young's inequality with exponents $(2,2)$, we obtain

$$
\begin{aligned}
& \int_{B_{R}} \xi^{2}\left|\Delta_{h} H_{\frac{q}{2}}(\nabla u)\right|^{2} d x \leq c_{3}(q, n)\|f\|_{B_{p, \infty}^{\alpha}\left(B^{\prime}\right)}\|\nabla u\|_{L^{q}\left(B^{\prime}\right)}|h|^{\alpha-1}+c_{4}(R, q) I_{1} \\
& \leq c_{3}(q, n)\|f\|_{B_{p, \infty}^{\alpha}\left(B^{\prime}\right)}\|\nabla u\|_{L^{q}\left(B^{\prime}\right)}|h|^{\alpha-1}+c_{7}(R, q, n)\|\nabla u\|_{L^{q}\left(B^{\prime}\right)}^{q / 2}\left(\int_{B_{R}} \xi^{2}\left|\Delta_{h} H_{\frac{q}{2}}(\nabla u)\right|^{2} d x\right)^{\frac{1}{2}} \\
& \leq c_{3}(q, n)\|f\|_{B_{p, \infty}^{\alpha}\left(B^{\prime}\right)}\|\nabla u\|_{L^{q}\left(B^{\prime}\right)}|h|^{\alpha-1}+\frac{1}{2} c_{8}(R, q, n)\|\nabla u\|_{L^{q}\left(B^{\prime}\right)}^{q}+\frac{1}{2} \int_{B_{R}} \xi^{2}\left|\Delta_{h} H_{\frac{q}{2}}(\nabla u)\right|^{2} d x .
\end{aligned}
$$

Reabsorbing the integral in the right-hand side of (4.7) by the left-hand side, we get

$$
\int_{B_{R}} \xi^{2}\left|\Delta_{h} H_{\frac{q}{2}}(\nabla u)\right|^{2} d x \leq c\left(\|f\|_{B_{p, \infty}^{\alpha}\left(B^{\prime}\right)}\|\nabla u\|_{L^{q}\left(B^{\prime}\right)}|h|^{\alpha-1}+\|\nabla u\|_{L^{q}\left(B^{\prime}\right)}^{q}\right),
$$

from which we can infer

$$
\int_{B_{R / 2}}\left|\Delta_{h} H_{\frac{q}{2}}(\nabla u)\right|^{2} d x \leq c\left(\|f\|_{B_{p, \infty}^{\alpha}\left(B^{\prime}\right)}\|\nabla u\|_{L^{q}\left(B^{\prime}\right)}|h|^{\alpha-1}+\|\nabla u\|_{L^{q}\left(B^{\prime}\right)}^{q}\right),
$$

with $c=c(R, q, n)>0$. Multiplying both sides of (4.8) by $|h|^{1-\alpha}$, we then have

$$
\int_{B_{R / 2}}\left|\frac{\tau_{h} H_{q / 2}(\nabla u)-H_{q / 2}(\nabla u)}{|h|^{(\alpha+1) / 2}}\right|^{2} d x \leq c\left(\|f\|_{B_{p, \infty}^{\alpha}\left(B^{\prime}\right)}\|\nabla u\|_{L^{q}\left(B^{\prime}\right)}+\|\nabla u\|_{L^{q}\left(B^{\prime}\right)}^{q}|h|^{1-\alpha}\right) .
$$

Since the above estimate holds for every $h \in \mathbb{R} \backslash\{0\}$ such that $|h| \leq r_{0}$, we can take the supremum over $|h|<\delta$ for some $\delta<r_{0}$ and obtain

$$
\sup _{|h|<\delta} \int_{B_{R / 2}}\left|\frac{\tau_{h} H_{q / 2}(\nabla u)-H_{q / 2}(\nabla u)}{|h|^{(\alpha+1) / 2}}\right|^{2} d x \leq c\left(\|f\|_{B_{p, \infty}^{\alpha}\left(B^{\prime}\right)}\|\nabla u\|_{L^{q}\left(B^{\prime}\right)}+\|\nabla u\|_{L^{q}\left(B^{\prime}\right)}^{q} r_{0}^{1-\alpha}\right),
$$

which gives the desired conclusion. 
The Besov regularity of $H_{\frac{q}{2}}(\nabla u)$ established in the previous theorem allows us to get a gain of integrability for $\nabla u$. More precisely, we have the following

Corollary 4.2. Under the assumptions of Theorem 4.1, we get

$$
H_{\frac{q}{2}}(\nabla u) \in L_{l o c}^{r}(\Omega) \text { for all } r \in\left[1, \frac{2 n}{n-\alpha-1}\right)
$$

and

$$
\nabla u \in L_{l o c}^{s}(\Omega) \text { for all } s \in\left[1, \frac{n q}{n-\alpha-1}\right)
$$

Proof. By observing that $\alpha+1<n$, from Theorems 4.1 and 2.10 we immediately obtain the first conclusion. Then

$$
\int_{K}(|\nabla u(x)|-1)_{+}^{\frac{q r}{2}} d x=\int_{K}\left|H_{\frac{q}{2}}(\nabla u(x))\right|^{r} d x<+\infty
$$

for all $r \in\left[1, \frac{2 n}{n-\alpha-1}\right)$ and all compact subsets $K$ of $\Omega$, which ensures that $\nabla u \in L_{l o c}^{s}(\Omega)$ for all $s \in\left[1, \frac{n q}{n-\alpha-1}\right)$.

Remark 4.3. Let us observe that when $n=2$ (the case which is relevant for applications to network congestion), the previous result implies that $\nabla u \in L_{l o c}^{s}(\Omega)$ for all $s<2 q /(1-\alpha)$. This means that for every $r>2 q$ there exists a $\delta=\delta(r, q) \in(0,1)$ such that, if $\alpha \in(\delta, 1)$ and $f \in B_{p, \infty, l o c}^{\alpha}(\Omega)$, then $\nabla u \in L_{l o c}^{r}(\Omega)$. In fact, it is enough to take $\delta=1-2 q / r$. In other words, given any $r>2 q$, we can choose $\alpha$ close enough to 1 in order to have $\nabla u \in L_{l o c}^{r}(\Omega)$ whenever $f \in B_{p, \infty, l o c}^{\alpha}(\Omega)$.

Again, if we come back to the variational problem (ㅍ1), we obtain the following higher integrability result for its minimizer:

Corollary 4.4. Let $n \geq 2, q \geq 2, \alpha \in(0,1)$ and $f \in B_{p, \infty, l o c}^{\alpha}(\Omega)$. Moreover, let $\sigma_{0} \in L^{p}\left(\Omega, \mathbb{R}^{n}\right)$ be the solution of ( $(\underline{\mathrm{P} 1})$. Then $\sigma_{0} \in L_{l o c}^{r}(\Omega)$ for all $r \in\left[1, \frac{n p}{n-\alpha-1}\right)$.

Proof. By duality, we know that $\sigma_{0}$ is related to any solution $u$ of problem (P2) through the optimality condition

$$
\sigma_{0}(x)=\nabla \mathcal{H}^{*}(\nabla u(x))=(|\nabla u(x)|-1)_{+}^{q-1} \frac{\nabla u(x)}{|\nabla u(x)|}, \quad \text { for } \mathscr{L}^{n} \text {-a.e. } x \in \Omega .
$$

Since $u \in W^{1, q}(\Omega)$ is a weak solution of the Euler-Lagrange equation (1.2) and $\left|\sigma_{0}\right|=\left|H_{\frac{q}{2}}(\nabla u)\right|^{\frac{2}{p}}$ $\mathscr{L}^{n}$-a.e. in $\Omega$, the assertion immediately follows from Corollary 4.2 .

Remark 4.5. Let us observe that when $n=2$, the previous result implies that $\sigma_{0} \in L_{l o c}^{r}(\Omega)$ for all $r<2 p /(1-\alpha)$. This means that for every $s>2 p$ there exists a $\delta=\delta(s, p) \in(0,1)$ such that, if $\alpha \in(\delta, 1)$ and $f \in B_{p, \infty, l o c}^{\alpha}(\Omega)$, then $\sigma_{0} \in L_{l o c}^{s}(\Omega)$. In fact, it is enough to take $\delta=1-2 p / s$. In other words, given any $s>2 p$, we can choose $\alpha$ close enough to 1 in order to have $\sigma_{0} \in L_{l o c}^{s}(\Omega)$ whenever $f \in B_{p, \infty, l o c}^{\alpha}(\Omega)$. 
Acknowledgements. The author gratefully acknowledges fruitful discussions with Antonia Passarelli di Napoli during the preparation of this paper.

Moreover, he would like to thank the reviewer for his/her suggestions to improve this work.

\section{References}

[1] E. Acerbi, N. Fusco, Regularity for minimizers of non-quadratic functionals: the case $1<p<2$, J. Math. Anal. Appl., 140 (1989), 115-135.

[2] J.-B. Baillon, G. Carlier, From discrete to continuous Wardrop equilibria, Newt. Heterogenous Media, 7 (2012), 219-241.

[3] A. L. Baisón, A. Clop, R. Giova, J. Orobitg, A. Passarelli di Napoli, Fractional differentiability for solutions of nonlinear elliptic equations, Potential Anal. 46 (3), 403-430 (2017).

[4] A. K. Balci, L. Diening, M. Weimar, Higher order Calderón-Zygmund estimates for the p-Laplace equation, J. Differential Equations, 268 (2020), 590-635.

[5] M. J. Beckmann, A continuous model of transportation, Econometrica, 20 (1952), 643-660.

[6] L. Brasco, Global $L^{\infty}$ gradient estimates for solutions to a certain degenerate elliptic equation, Nonlinear Anal. 74 (2011), 516-531.

[7] L. Brasco, G. Carlier, Congested traffic equilibria and degenerate anisotropic PDEs, Dynamic Games and Applications, 3 (4): 508-522, 2013.

[8] L. Brasco, G. Carlier, On certain anisotropic elliptic equations arising in congested optimal transport: Local gradient bounds, Advances in Calculus of Variations 7, no. 3 (2014), 379407.

[9] L. Brasco, G. Carlier, F. Santambrogio, Congested traffic dynamics, weak flows and very degenerate elliptic equations, J. Math. Pures Appl., 93 (2010), 652-671.

[10] M. Chipot, L. C. Evans, Linearization at infinity and Lipschitz estimates for certain problems in the calculus of variations, Proc. Roy. Soc. Edinburgh Sect. A, 102 (1986), 291-303.

[11] A. Clop, R. Giova, F. Hatami, A. Passarelli di Napoli, Very degenerate elliptic equations under almost critical Sobolev regularity, Forum Mathematicum 32, no. 6 (2020): 1515-1537.

[12] A. Clop, R. Giova, A. Passarelli di Napoli, Besov regularity for solutions of p-harmonic equations, Adv. Nonlinear Anal. 2019; 8: 762-778.

[13] G. Cupini, F. Giannetti, R. Giova, A. Passarelli di Napoli, Regularity results for vectorial minimizers of a class of degenerate convex integrals, J. Differential Equations, 265 (2018), 4375-4416.

[14] I. Ekeland, R. Témam, Convex Analysis and Variational Problems, Classics in Applied Mathematics 28, SIAM, Philadelphia, 1999. 
[15] M. Eleuteri, P. Marcellini, E. Mascolo, Lipschitz estimates for systems with ellipticity conditions at infinity, Annali di Matematica Pura e Applicata (2016) 195: 1575-1603.

[16] M. Eleuteri, P. Marcellini, E. Mascolo, Regularity for scalar integrals without structure conditions, Advances in Calculus of Variations 13, no. 3 (2020): 279-300.

[17] I. Fonseca, N. Fusco, P. Marcellini, An existence result for a nonconvex variational problem via regularity, ESAIM: Control, Optimisation and Calculus of Variations, 7 (2002), 69-95.

[18] A. Gentile, Regularity for minimizers of a class of non-autonomous functionals with subquadratic growth, Advances in Calculus of Variations, 2020.

[19] M. Giaquinta, G. Modica, Remarks on the regularity of the minimizers of certain degenerate functionals, Manuscripta math. 57 (1986), 55-99.

[20] E. Giusti, Direct Methods in the Calculus of Variations, World Scientific Publishing Co., 2003.

[21] D. D. Haroske, Envelopes and Sharp Embeddings of Function Spaces, Chapman \& Hall CRC, 2006.

[22] C. Leone, A. Passarelli di Napoli, A. Verde, Lipschitz regularity for some asymptotically subquadratic problems, Nonlinear Anal. 67 (2007), 1532-1539.

[23] A. Passarelli di Napoli, A. Verde, A regularity result for asymptotically convex problems with lower order terms, J. Convex Anal. 15 (2008), No. 1, 131-148.

[24] J.P. Raymond, Lipschitz regularity of solutions of some asymptotically convex problems, Proc. Roy. Soc. Edinburgh Sect. A, 117 (1991), 59-73.

[25] L. Tartar, An Introduction to Sobolev Spaces and Interpolation Spaces, Lecture Notes of the Unione Mat. Italiana 3, Springer-Verlag Berlin Heidelberg, 2007.

[26] P. Tolksdorff, Everywhere-regularity for some quasilinear systems with a lack of ellipticity, Ann. Mat. Pura Appl. 134 (1983), 241-266.

[27] H. Triebel, Theory of Function Spaces, Monogr. Math. 78, Birkhäuser, Basel, 1983.

\section{Pasquale Ambrosio}

Dipartimento di Matematica e Applicazioni "R. Caccioppoli"

Università degli Studi di Napoli "Federico II"

Via Cintia, 80126 Napoli, Italy.

E-mail address: pasquale.ambrosio2@studenti.unina.it 